\title{
Quantitative assessment based on kinematic measures of functional impairments during upper extremity movements: A review
}

\author{
Ana de los Reyes-Guzmán ，Iris Dimbwadyo-Terrer , Fernando Trincado-Alonso ,Félix Monasterio-Huelin \\ Diego Torricelli , Angel Gil-Agudo
}

\begin{abstract}
A B S T R A C T
Background: Quantitative measures of human movement quality are important for discriminating healthy and pathological conditions and for expressing the outcomes and clinically important changes in subjects' functional state. However the most frequently used instruments for the upper extremity functional assessment are clinical scales, that previously have been standardized and validated, but have a high subjective component depending on the observer who scores the test. But they are not enough to assess motor strategies used during movements, and their use in combination with other more objective measures is necessary. The objective of the present review is to provide an overview on objective metrics found in literature with the aim of quantifying the upper extremity performance during functional tasks, regardless of the equipment or system used for registering kinematic data.

Methods: A search in Medline, Google Scholar and IEEE Xplore databases was performed following a combination of a series of keywords. The full scientific papers that fulfilled the inclusion criteria were included in the review. Findings: A set of kinematic metrics was found in literature in relation to joint displacements, analysis of hand trajectories and velocity profiles. These metrics were classified into different categories according to the movement characteristic that was being measured.

Interpretation: These kinematic metrics provide the starting point for a proposed objective metrics for the functional assessment of the upper extremity in people with movement disorders as a consequence of neurological injuries. Potential areas of future and further research are presented in the Discussion section.
\end{abstract}

\section{Introduction}

Impaired upper extremity function is one of the most common sequelae after Central Nervous System (CNS) injuries (Nakayama et al., 1994; Parker et al., 1986; Wade, 1992). Quantitative measures of human movement quality are significant in the rehabilitation field for expressing the outcomes during rehabilitation treatments and assessing their efficacy, discriminating between healthy and pathological conditions (Yang et al., 2002), and for helping in the decision making in the clinical setting.

The most frequently used measure instruments in clinical settings for the upper extremity assessment are a set of classic clinical scales, which have been previously defined, validated and standardized. These scales are easy to administer, however, the main disadvantage of these quantitative measures is that they have a high subjective component, depending on the observer who visually scores the test. These clinical scales have been classified into several categories such as strength, functional or activities of daily living (ADLs) tests (Van Tuijl et al., 2002). The functional tests are classified into general clinical scales such as Jebsen-Taylor Hand Function (Jebsen et al., 1969), Arm Research Assessment Test (ARAT) and Nine-Hole Peg Test; and specific clinical scales applied to spinal cord injury (SCI) (Van Tuijl et al., 2002), stroke (Fugl-Meyer et al., 1974) or cerebral palsy (CP) (Boyce et al., 1991). Related to the ADL assessment some of the most important measures are Barthel Index (Mahoney, 1965) or Functional Independence Measure (FIM) (Keith, 1987) and specific in SCI, Spinal Cord Independence Measure (SCIM) (Catz et al., 1997), but still having a very high subjective component depending on the observer who scores the test. However, only functional and clinical assessments are not enough to assess motor strategies used during movements (Cacho et al., 2011), and their use in combination with other more objective measures is necessary.

The idea of using objective metrics for the upper extremity assessment isn't new. The reaching movement has been analyzed for more 
than 100 years. Burdet et al. proposed that the first experience was performed by Woodworth in 1899 for quantifying reaching movements (Burdet and Milner, 1998). Early, in 1954, Fitts proposed two objective metrics for quantifying the performance and the difficulty of a task. For this purpose, in the experiments performed, the movement amplitude and the size of the object to reach were modified with the aim of analyzing the speed-accuracy trade-off during a task (Fitts, 1954). This trade-off has been studied by other authors. Even Fitts' metrics were extended and two modified metrics were proposed for the assessment of the movement quality (Yang et al., 2002). Burdet showed that the velocity profile of adult subjects depends on accuracy requirements during the task (Burdet and Milner, 1998) and Volman analyzed how the functional context of the task influenced the motor performance which shows the results in term of kinematic metrics (Volman et al., 2002).

The kinematic study can provide accurate and objective information about motor strategies associated with goal-oriented tasks, and monitor administration of therapeutic techniques for the upper extremity. During the last fifteen years, many kinematic studies have been performed in laboratory settings with the aim of quantifying the upper extremity movements during complete ADL in healthy people (Aizawa et al., 2010; Magermans et al., 2005; Murgia et al., 2010; Murphy et al., 2006; Namdari et al., 2012; Petuskey et al., 2007; Van Andel et al., 2008) and people who have suffered stroke (Kim et al., 2014; Lang et al., 2006; Murphy et al., 2011, 2013; Osu et al., 2011), CP (Butler et al., 2010; Klotz et al., 2013) or SCI (de los Reyes-Guzmán et al., 2010). An important component of a rehabilitation treatment is a quantified assessment that is sensitive to the outcome of interest. Murphy et al. have studied several kinematic metrics responsiveness during the ADL of drinking and the results have been compared with clinical scales (Murphy et al., 2013) to identify motor and functional recovery.

On the other hand, devices for robot-aided neurorehabilitation are increasingly being incorporated into patients' care programs after stroke (Bosecker et al., 2010; Colombo et al., 2008, 2012; Ellis et al., 2008; Zollo et al., 2011) or spinal cord injury (Zariffa et al., 2012), and these devices can serve as precise and reliable measurement tools that record simultaneously kinematic and kinetic data. Recently, Bosecker et al. $(2010)$ and Colombo et al. $(2008,2012)$ have provided the first experiences of evaluation metrics based on robot kinematic data for quantifying motor recovery of stroke patients during robot-aided rehabilitation. Although a previous review was published related to metrics from data measured by means of robotic systems (Balasubramanian et al., 2012a), to our knowledge, a survey was not found related to objective metrics regardless of the system used for extracting kinematics data. Kinematic data are a quantified measure of active and passive ranges of movement, the size of the workspace of the hand, the execution time, and the velocity of the movement. Kinematic also provides information about the movement's quality with respect to coordination, smoothness and other functional characteristics (Murphy et al., 2011).

The aim of the present review is to present an overview in relation to objective metrics found in literature to quantify the upper extremity function regardless of the system used for extracting kinematic data and the movements analyzed. Firstly, the methodology related to the search strategy and the inclusion and exclusion criteria for selecting articles is presented. In the Results section, the objective metrics found in literature are described and classified according to the movement characteristic that they quantify. Then potential aspects for further research are discussed.

\section{Methods}

\subsection{Search strategy}

An electronic search was performed by one reviewer to find all articles on the topic of functional assessment of the upper limb. The databases included Medline, Google Scholar and IEEE Xplore covering the period from 2002 to December 2013, using combinations of the keywords "upper extremity", "functional assessment", "kinematics", "robotic rehabilitation", "movement quality" and "objective metrics". "And" and "Or" conjunctions were used during the search. Only English language articles were considered. A manual search of references of relevant considered studies and other publications from the authors of the found articles was also performed.

\subsection{Inclusion and exclusion criteria}

Three people assessed the titles and abstract of the articles. The articles included in the study fulfill the following criteria: (i) investigation of the upper extremity during reaching movements, activities of daily living or functional tasks, (ii) clear and documented purpose of the definition or application of quantitative functional metrics from kinematic data, (iii) full scientific papers. Reports related to robotics systems were included if the purpose of the study was the quantitative assessment from kinematic data. Studies with the aim of quantifying the motor performance of the upper extremity from kinematics data in healthy people or after neurological injuries such as stroke, cerebral palsy or spinal cord injury were included. Studies published as conference proceedings were excluded from the review.

\section{Results}

\subsection{Search yield}

The initial search of the databases yielded 737 results. After the review of titles and abstracts and to reject duplicated articles, 141 articles were selected. After the application of the inclusion and exclusion criteria, 40 articles related with the functional evaluation of the upper limb in an objective way were selected and other 5 articles related to the upper extremity quantitative assessment from kinematic data were identified from the manual targeted search. A total of 45 articles were selected for inclusion in the current review (Fig. 1).

In the following sections, the term reviewed articles is related to the 45 selected articles.

\subsection{Movement analyzed}

The upper extremity movements were classified into two categories according to clinical scale classification: functional movements and complete ADL (Table 1) (Van Tuijl et al., 2002). This method was used in the following sections for classifying the 45 selected articles.

During the last 10 years, several kinematic studies have focused on ADL involving an upper extremity (Aizawa et al., 2010; Butler et al., 2010; de los Reyes-Guzmán et al., 2010; Magermans et al., 2005; Murphy et al., 2006; Namdari et al., 2012; Petuskey et al., 2007). Kinematic data for upper extremities can be collected using studies of various tasks considered representative of ADL. Furthermore, purposeoriented movements must be analyzed because the musculoskeletal system can accomplish a motor task in many ways by selecting a suitable trajectory and inter-joint coordination (Kim et al., 2014) and it provides more validity to the studies performed (Murphy et al., 2011).

Within the populations analyzed, in healthy people the most common movement analyzed was ADL, in $7(77.8 \%)$ of the studies in this population. Normally, these studies analyzed several ADLs with the aim of computing normative data in relation to kinematic variables (Aizawa et al., 2010; Murgia et al., 2010; Murphy et al., 2006; Namdari et al., 2012). However, in populations with neurological injury, the most frequently analyzed movements were functional movements related to reaching movements, reach and grasp movements or drawing trajectories as circles or squares (Tables 1 and 2). 


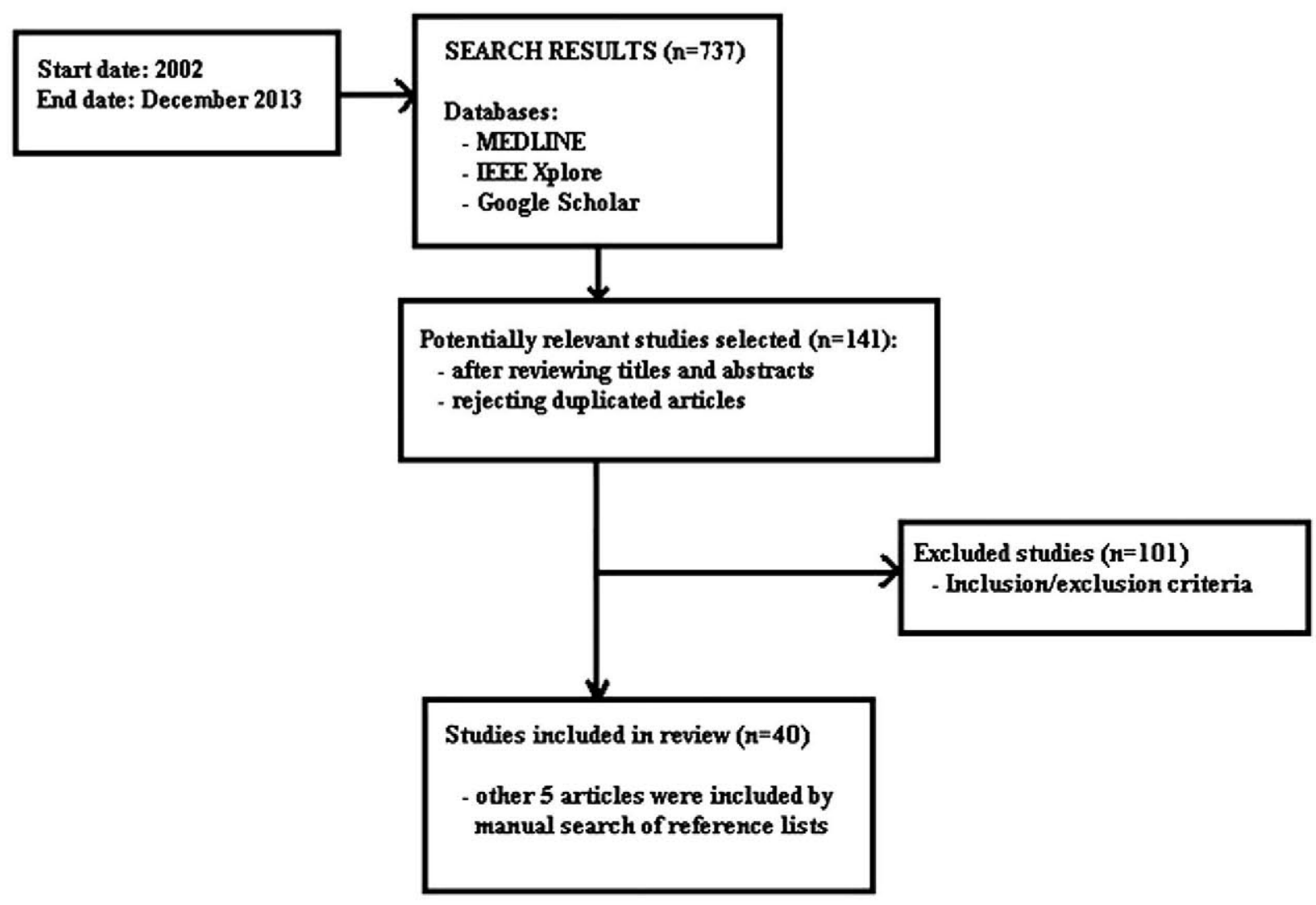

Fig. 1. Flow diagram of the search strategy results.

\subsubsection{Movement capture systems}

In the reviewed articles, the motion capture techniques most frequently used were opto-electronic systems based on active or passive markers. 24 (53.4\%) of the reviewed studies have used these systems (Table 2). Robotic devices were the following most frequently used, in $15(33.3 \%)$ of the total full articles; 5 studies (11.1\%) used electromagnetic sensors for registering the upper extremity movement; and only one study used inertial sensors $(2.2 \%)$. These results have been included in Table 2 .

\subsection{CNS injuries}

Usually kinematic metrics have been used for quantifying the motor performance of healthy people, or people who have suffered any neurological injury, such as stroke, $\mathrm{SCI}$ or $\mathrm{CP}$ with the aim of finding significant differences between the groups analyzed for the metrics proposed. As it has been shown in a previous section, full articles related to the functional assessment with objective metrics in people with any of these injuries were included for reviewing (Table 2).

The neurological injury most frequently studied was stroke, in 25 (55.6\%) of the reviewed articles; CP was analyzed in $8(17.7 \%)$ of the selected studies and 3 studies, the $6.7 \%$, corresponded to metrics application in people with SCI. The rest of the articles, 9 studies (20\%), were related to healthy population.

\subsection{Purpose of the metrics}

Measure instruments must be designed according to the purpose of the measure for using in a clinical setting. Several of the selected articles performed experimental trials with healthy people and people who have suffered stroke, CP or SCI (Table 2). These studies applied kinematic metrics with any of these purposes:

- Discriminative measures: Metrics are applied with the aim of detecting statistical significant differences between healthy people and people with neurological injury during the performance of functional movements (Jaspers et al., 2011; Van der Heide et al., 2005) and ADL (de los Reyes-Guzmán et al., 2010; Murphy et al., 2011).

- Evaluative measures: Metrics could be useful as evaluative measure if they fulfill the properties of validity, reproducibility and sensitivity to the change:

O Validity: Kinematic metrics must correlate highly with clinical scales (Celik et al., 2010; Colombo et al., 2008; Osu et al., 2011; Van der Heide et al., 2005).

O Reproducibility: Metrics must present consistency between repeated measures within the session and between consecutive days in the absence of clinical changes in a subject functional ability (de los Reyes-Guzmán et al., 2010; Osu et al., 2011; Wagner et al., 2008).

O Sensitivity to the change: Metrics must have the ability of detecting changes if any clinical change (positive or negative) has occurred in relation to the functional ability of a subject (Murphy et al., 2013).

- Predictive measures: Metrics are used for predicting the outcomes in clinical scales by the application of regression models (Zariffa et al., 2012).

\subsection{Upper extremity movement-related metrics}

In this section, a set of movement-related metrics has been proposed through the review performed. These kinematic metrics were obtained 
Table 1

Three categories for classifying the upper extremity movements analyzed.

\begin{tabular}{|c|c|c|}
\hline Movement category & Definition & Examples \\
\hline \multicolumn{3}{|l|}{ Functional movements } \\
\hline Reaching movements & Point to point movements with starting and ending locations & $\begin{array}{l}\text { Point to point movements; reaching movements (horizontal and vertical } \\
\text { planes); reach and grasp movements }\end{array}$ \\
\hline Path drawing & $\begin{array}{l}\text { Movements which require follow a desired closed trajectory. The ending } \\
\text { point corresponds with the starting point }\end{array}$ & Drawing a circle path; a square path; figure-of- 8 trajectory \\
\hline ADL & $\begin{array}{l}\text { Basic activities of daily living which involve reaching movement, object } \\
\text { manipulation, proximal and distal transport movements, releasing the } \\
\text { object and return to the starting position }\end{array}$ & $\begin{array}{l}\text { Drinking from a glass; to move the hand to the mouth; to move the hand to } \\
\text { the head for combing; personal hygiene }\end{array}$ \\
\hline
\end{tabular}

from measurements performed through the opto-electronic systems and devices included in Table 2 .

Each of the movement metrics examines a specific aspect of motor ability or movement characteristic (Figs. 2a and 2b). In a previous review on metrics from the use of robotic systems, Zollo et al. noted that the information provided by each of these measures may not be exclusive, and the names used in their survey may differ from those used in the original and cited papers (Zollo et al., 2011). In the present review, we highlighted the same difficulty. The same metrics were called with different names and so the adopted criteria for presenting the metrics found in literature to the date were to use the most recently proposed name in the reviewed literature.

The objective metrics proposed have been computed from joint displacements, the trajectory or the speed or acceleration profile of the hand during the movement analyzed, and metrics have been classified into 9 groups according to the movement characteristic that it tries to quantify.

\subsubsection{Neuromuscular capability (functional range of motion)}

Two metrics were classified into this group and quantify the upper extremity range of motion from joints (Fig. 2b) and hand position data (Fig. 2a).

\section{- Joint range of motion}

Within a given task this metric is a measure of the joint displacement (Fig. 2b). This metric was computed during functional movements (Ellis et al., 2008; Jaspers et al., 2011; Rönnqvist and Rösblad, 2007) and complete ADL (Aizawa et al., 2010; de los Reyes-Guzmán et al., 2010; Fitoussi et al., 2006; Kim et al., 2014; Murgia et al., 2010; Murphy et al., 2006, 2011; Namdari et al., 2012; Petuskey et al., 2007; Van Andel et al., 2008). This metric was expressed in degrees.
- Reaching range of motion

Related to the hand displacement during a task, it was computed in Cartesian coordinates $\mathrm{x}, \mathrm{y}, \mathrm{z}$ during reaching movements (Zariffa et al., 2012). Balasubramanian et al. defined the reachable workspace as the region that can be reached by the subject voluntarily (Balasubramanian et al., 2012a) (Fig. 2a).

\subsubsection{Movement speed}

Two metrics were classified into this movement characteristic:

\section{- Movement time}

It's a measure of the time required to perform a functional task successfully and quantifies the movement speed (Fig. 2a). This metric was applied in practically all the studies reviewed. An improvement in this metric is attributed to a better upper extremity function within a given task. In reach and grasp movements, two modalities of movement time could be estimated: (i) the movement time related to reach the object to manipulate and (ii) the movement time required to stabilize the arm around the target and grasping the object (Balasubramanian et al., 2012a). This metric applied to functional movements mostly reaching movements, was used by several authors (Balasubramanian et al., 2012a; Chang et al., 2005; Fasoli et al., 2002; Jaspers et al., 2011; Van der Heide et al., 2005; Volman et al., 2002) and writing trajectories (Culmer et al., 2009).

In the analysis of ADL, the total movement time was divided into phases composing the complete ADL. For example, in the activity of drinking from a glass, the movement was divided into five phases and events delimiting the phases: reaching (included grasping), forward transport, drinking, back transport (included releasing the object) and returning to the starting point. In these cases, the total movement time was computed by summing the movement time

Table 2

Classification of the reviewed articles by motion capture system used and movements analyzed.

\begin{tabular}{|c|c|c|c|c|c|}
\hline & Healthy $(n=9)$ & Stroke $(n=25)$ & $\mathrm{CP}^{\prime}(\mathrm{n}=8)$ & $\mathrm{SCl}(\mathrm{n}=3)$ & Total reviewed articles (\%) \\
\hline \multicolumn{6}{|l|}{ Motion capture system } \\
\hline Opto-electronic system & $5^{[4,17,19,20,21]}$ & $10^{[23-27,41,45,50,52]}$ & $7^{[28,29,38,39,42-44]}$ & $2^{[12,30]}$ & 53.4 \\
\hline Robotic device & $1^{[47]}$ & $12^{[31-35,40,46,53-55,57,61]}$ & $1^{[15]}$ & $1^{[36]}$ & 11.1 \\
\hline Electromagnetic sensors & $3^{[16,18,22]}$ & $2^{[51,59]}$ & - & - & 2.2 \\
\hline Inertial sensors & - & $1^{[58]}$ & - & - & 33.3 \\
\hline \multicolumn{6}{|l|}{ Movement analyzed } \\
\hline \multicolumn{6}{|l|}{ Functional movements } \\
\hline Reaching movements & $2^{\mid 4,47]}$ & $15^{[27,31-35,40,41,45,49-52,54,55]}$ & $6^{[15,38,39,42-44]}$ & $2^{[12,36]}$ & 55.6 \\
\hline Path drawing & - & $4^{[46,53,57,61]}$ & - & - & 8.9 \\
\hline ADL & $7^{[16-21]}$ & $6^{[23-26,58,59]}$ & $2^{[28,29]}$ & $1^{[30]}$ & 35.5 \\
\hline
\end{tabular}

The numbers included between brackets are related to the following studies included in the bibliography:

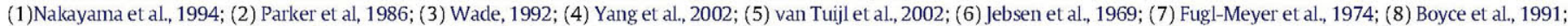

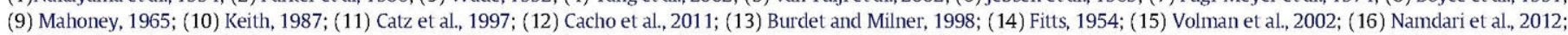

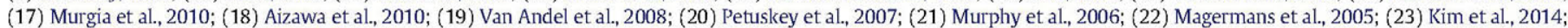

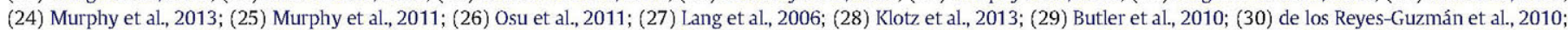

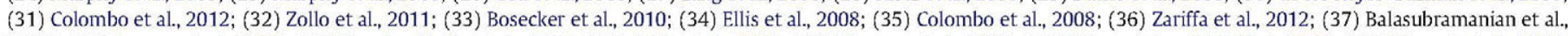

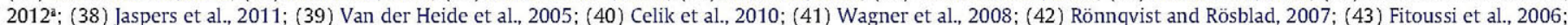

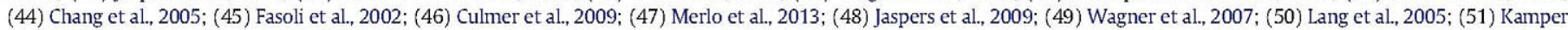

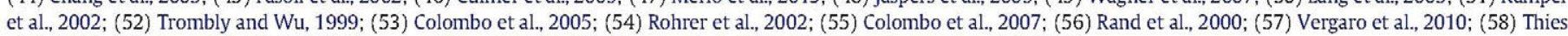
et al., 2009; (59) Lum et al., 2009; (60) Hogan and Sternad, 2009; (61) Dipietro et al., 2007; (62) Balasubramanian et al., $2012 b$. 


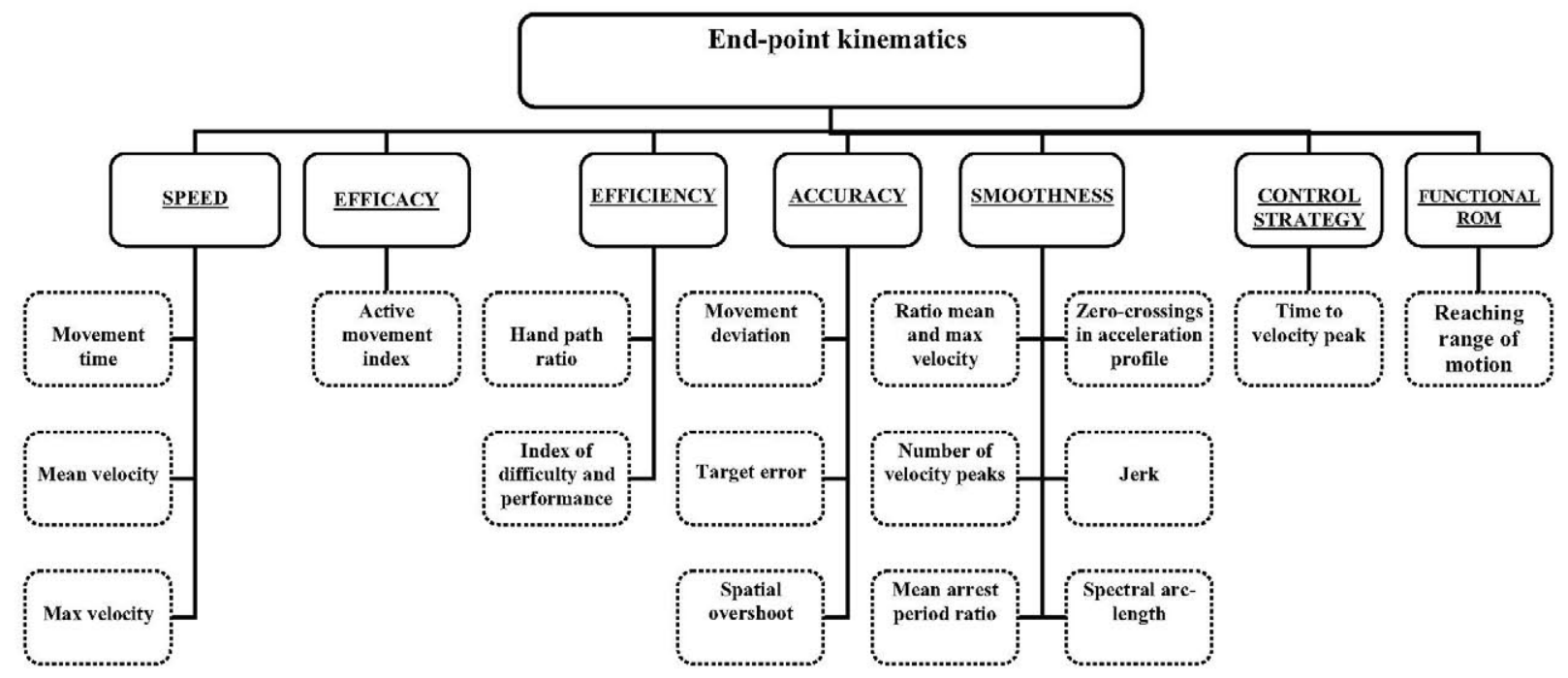

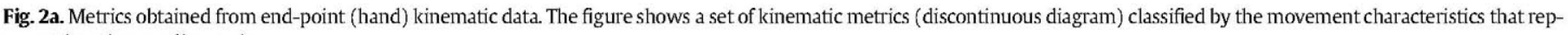
resent (continuous diagram).

of each movement phase (de los Reyes-Guzmán et al., 2010; Murphy et al., 2011). The results were expressed in seconds and as a percentage of the total movement cycle. Other studies applied this metric to complete ADL (Butler et al., 2010; Murphy et al., 2006, 2013).

- Maximum and mean velocity

These measures are related to the arm velocity attained during the complete movement and they were computed from speed profile of the hand or the end-effector (Balasubramanian et al., 2012a; Merlo et al., 2013). The most frequently used parameter was the maximum hand velocity (Butler et al., 2010; Cacho et al., 2011; de los Reyes-Guzmán et al., 2010; Fasoli et al., 2002; Jaspers et al., 2009; Kamper et al., 2002; Lang et al., 2005, 2006; Murphy et al., 2006, 2011; Trombly and Wu, 1999; Van der Heide et al., 2005; Volman et al., 2002; Wagner et al., 2007, 2008). Other studies analyzed only the mean hand velocity during the movement (Bosecker et al., 2010; Celik et al., 2010; Colombo et al., 2005, 2012; Rohrer et al., 2002; Zariffa et al., 2012).

People with movement disorders after neurological injury perform the movements with several peaks in the hand velocity profile. Usually, the first velocity peak and the maximum peak aren't the same, so Murphy proposed to analyze the first velocity peak with the aim of quantifying the movement initial effort (Murphy et al., 2011). In relation to the movements analyzed, reaching movement was the most frequently analyzed movement, so these values were computed between the starting point and reaching the object (Balasubramanian et al., 2012a; Butler et al., 2010; Cacho et al., 2011; Celik et al., 2010; Colombo et al., 2012; Fasoli et al., 2002; Jaspers et al., 2009; Kamper et al., 2002; Lang et al., 2005, 2006; Merlo et al., 2013; Rohrer et al., 2002; Trombly and Wu, 1999; Van der Heide et al., 2005; Volman et al., 2002; Wagner et al., 2007, 2008; Zariffa et al., 2012) or during hand drawing trajectories (Bosecker et al., 2010; Colombo et al., 2005) but they were representative for analyzing ADLs (de los Reyes-Guzmán et al., 2010; Murphy et al., 2011).

\subsubsection{Movement efficacy}

\section{- Active movement index}

Within a given task this metric is defined as the percentage of the task that the subject has performed voluntarily in an active way. It's a measure of the movement efficacy (Colombo et al., 2007, 2008, 2012) (Fig. 2a). It's worth to take into account that this metric was usually computed from the use of robotic devices, in which a person starts the movement in a voluntary way and then, if there isn't any movement, the robotic device assists the movement during the task performance. However, this metric was called Fraction of Reach (FOR) during reaching movements and was computed from spatial coordinates by means of electromagnetic sensors (Kamper et al., 2002).

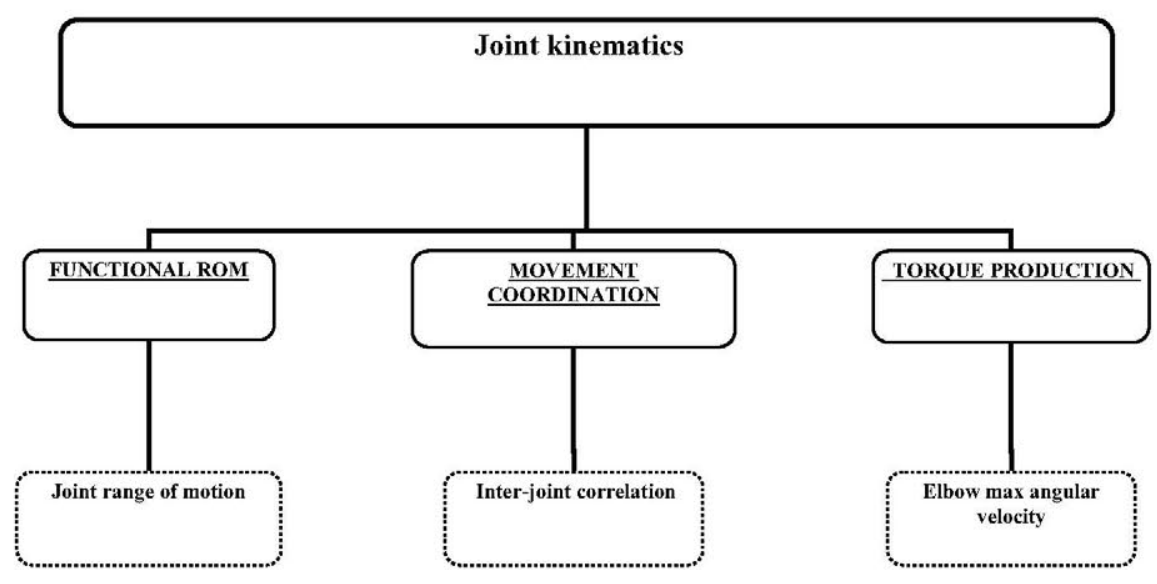

Fig. 2b. Metrics obtained from joint kinematic data. The figure shows a set of kinematic metrics (discontinuous diagram) classified by the movement characteristics that represent (continuous diagram). 


\subsubsection{Movement efficiency}

- Hand path ratio

It's a measure of how directly the hand moves toward the target computed as the ratio between the length of the real subject's hand path and the length of the theoretical or desired trajectory. This metric quantifies the movement efficiency (Fig. 2a). Lang defined an efficient movement as the movement that moves directly to the target without extraneous or abnormal trajectories (Lang et al., 2005).

This metric has been frequently used in literature during reaching movements (Cacho et al., 2011; Jaspers et al., 2011; Kamper et al., 2002; Lang et al., 2005; Merlo et al., 2013; Van der Heide et al., 2005; Wagner et al., 2008). The reaching movement in healthy people is a highly stereotypic and coordinated movement consisting of a well-executing arm trajectory (Rand et al., 2000). The theoretical movement trajectory was considered the straight line between the starting point and the target location (Butler et al., 2010; Cacho et al., 2011; Culmer et al., 2009; Jaspers et al., 2011; Kamper et al., 2002; Lang et al., 2005, 2006; Merlo et al., 2013; Van der Heide et al., 2005). So a metric result closed to 1 is representative of a healthy pattern (Colombo et al., 2008). Patients tend to perform more curved trajectories resulting in values greater than 1 , so an increase of this metric is related to a longer hand trajectory during the movement. Other studies showed this result as a percentage (Merlo et al., 2013) and called this metric directness of the movement (Trombly and Wu, 1999), direct efficiency (Colombo et al., 2008) or index of curvature (Cacho et al., 2011; Jaspers et al., 2011; Van der Heide et al., 2005).

In literature, one study was found that computed the trajectory curvature from the first and second time derivatives of hand position data within an ADL (Osu et al., 2011).

- Index of difficulty and performance

These metrics are measures of the movement quality computed from the time required to perform a reaching movement, the distance between the start and end points and the size of the object to reach to (Fitts, 1954). Later, Yang et al. proposed the same quality metrics in terms of polar coordinates applied to reaching movements (Yang et al., 2002).

\subsubsection{Movement accuracy}

Three metrics were found within this classification: movement deviation between trajectories, the error around the target and the spatial overshoot.

\section{- Movement deviation}

It's a measure of the movement quality in terms of deviation of a subject's movement from a theoretical or desired trajectory (Balasubramanian et al., 2012a).

This metric is considered a measure of the error in accuracy (Colombo et al., 2008). In the literature, this metric was also called by the name of the movement characteristic that it represents "accuracy" (Colombo et al., 2008) (Fig. 2a) or "tracking error" (Vergaro et al., 2010). It's computed as the mean distance from the Euclidean distance between points of the real and theoretical hand trajectories (Celik et al., 2010; Colombo et al., 2007, 2008, 2012). Applied to reaching movements, the theoretical trajectory has been the straight line between start and end points.

Moreover the spatial deviation, the time variability of the trajectory was analyzed in the study performed by Thies et al. (2009).

- Target error

It's a measure of the movement quality in terms of accuracy (Fig. 2a) as the end-point error around the target placement. This metric was computed as the maximum distance from the index finger to the target location at the end of the movement (Lang et al., 2005). Target error was analyzed mostly in point to point or reach and grasp movements. This metric was called end-point error (Lang et al., 2005) in literature.

- Spatial overshoot

It's a measure of the spatial excess, if occurs, in any direction of the movement, out of the limits described by the starting and the target location and quantifies movement accuracy (Fig. 2a). This metric was proposed in literature computed in horizontal and vertical movement directions during reaching movements from endeffector position data (Merlo et al., 2013).

\subsubsection{Movement smoothness}

- Ratio between mean and maximum velocity

It's a measure of the ratio between both anterior metrics previously described as a quality measure in terms of smoothness (Fig. 2a). In a healthy subject this value should be close to one, but in the presence of movement disorders this metric could detect alterations during the movement pattern related to various acceleration and deceleration periods or the presence of brisk movements (Merlo et al., 2013). Several studies proposed this metric during reaching movements (Bosecker et al., 2010; Merlo et al., 2013; Rohrer et al., 2002; Zariffa et al., 2012) and path drawing (Bosecker et al., 2010).

- Number of velocity peaks

It's a quality measure of the movement smoothness computed from the speed profile of the movement hand (Fig. 2a). This metric was applied frequently in literature during reaching movements (Cacho et al., 2011; Chang et al., 2005; Fasoli et al., 2002; Merlo et al., 2013; Rohrer et al., 2002) and ADL (Butler et al., 2010; Murphy et al., 2011, 2013). Peak number was called movement units by several authors (Murphy et al., 2011, 2013; Rohrer et al., 2002).

A normal reaching movement has only one peak in the velocity profile of the hand movement (Chang et al., 2005). With the presence of movement disorders, the velocity peak number increases resulting in a less smooth movement. If any motor recovery occurs, the velocity profile of the hand movement must present less peaks resulting in a smoother movement (Rohrer et al., 2002).

- Mean arrest period ratio

This metric is related to the movement smoothness. In people with movement disorders, while performing a movement toward an objective, several stops usually occur. This produces a movement with several submovements with several periods of practically zero velocity (Rohrer et al., 2002). With recovery, a movement has less unnecessary stops and therefore the movement is smoother.

Recently, this metric has been computed as the ratio between the movement time in which the hand stops and the total movement time. The hand was considered "stopped" if the hand velocity was less than $20 \%$ of the mean velocity during the movement analyzed (Vergaro et al., 2010).

- Zero-crossings in acceleration profile

It measures the frequency of base line crossings in acceleration profile during the movement analyzed. This is a metric of the movement quality smoothness not usually used in literature. Only two articles have been found that proposed this metric during reaching movements (Aizawa et al., 2010; Trombly and Wu, 1999) and one during ADL (Lum et al., 2009).

- Jerk

The jerk metric represents the rate of change of the acceleration profile during a movement (Rohrer et al., 2002). This is a measure computed from the third time derivative of position during the hand movement and represents a measure of non-smoothness quality characteristic. Hogan proposed a set of jerk metrics (Hogan and Sternad, 2009). These measures, even those including a normalization factor (Chang et al., 2005; Culmer et al., 2009; Hogan and Sternad, 2009; Merlo et al., 2013; Rohrer et al., 2002) depended on the movement amplitude and the time. So these measures weren't dimensionless (Hogan and Sternad, 2009). 
- Spectral arc-length

This metric is related to the movement smoothness (Fig. 2a). As smooth movements are composed of low frequency components and a non-smooth movement is composed of higher frequency components, the use of Fourier Transform is adequate for the analysis of movement smoothness (Balasubramanian et al., 2012b). To our knowledge, this metric has been proposed only for an author that proposes the spectral arc-length metric as a measure and it's computed from the amplitude and Fourier magnitude spectrum from the velocity profile of the hand movement (Balasubramanian et al., $2012 \mathrm{~b}$ ) as the metrics related to smoothness previously described.

\subsubsection{Movement coordination}

- Inter-joint correlation

This metric has sense in arm movements which involve several joints resulting in a coordinated movement. Until now, it has been computed during reaching movements for analyzing the coordination between the shoulder and elbow flexion-extension movements. The method used to calculate this correlation depends on the sense of the movement analyzed. So in a reaching movement there is an almost linear relation between the shoulder and elbow joint displacements in the flexion-extension movements (de los Reyes-Guzmán et al., 2010; Dipietro et al., 2007; Murphy et al., 2006, 2011; Wagner et al., 2008) and the Pearson Correlation Index was used. Lum et al. have introduced the computing of this metric during the transport phase within an ADL. In this case, the index of correlation is computed from joint displacements of elbow flexion-extension and shoulder abduction-adduction (Lum et al., 2009).

Results in relation to this metric closed to 1 indicate a high correlation or relation between the variables analyzed and a high joint coordination.

\subsubsection{Movement control strategy}

- Time to velocity peak

A well-executed reaching movement is composed of only one velocity peak with a symmetrical wave form. One peak velocity displaced to the left is related to large deceleration periods and displaced to the right is related to ballistic or disrupted movements (Trombly and $\mathrm{Wu}, 1999$ ). Both situations have been identified with modified patterns with respect to a healthy motor performance. In the presence of movement disorders, reach and grasp movements may occur to be sequentially planned, with large deceleration periods and leftshifted velocity peaks. So it's a measure of the control strategy used during the movement (Fig. 2a).

In the articles reviewed, this measure was expressed in seconds and as a percentage of the reaching phase in which it occurs and was analyzed in several studies (Butler et al., 2010; Culmer et al., 2009; de los Reyes-Guzmán et al., 2010; Fasoli et al., 2002; Jaspers et al., 2009, 2011; Murphy et al., 2006; Trombly and Wu, 1999; Volman et al., 2002).

\subsubsection{Torque production}

- Elbow maximum angular velocity

This metric is computed by differentiation from elbow angular data and it's a measure of torque production (Chang et al., 2005) during a motor task (Fig. 2b). This measure has been used in the study performed by Murphy et al. (2011).

\section{Discussion}

This paper provides a review of the literature in the rehabilitation field in relation to quantitative metrics or variables, which have been proposed with the aim of assessing the quality and performance of the upper extremity movements. The quantification of the upper extremity function can be useful for analyzing the results of treatments and assessing their efficacy, discriminating between healthy and pathology conditions in relation to detect functional impairments, and for helping in the decision making in the clinical setting.

In literature, one review has been found in relation to kinematic metrics applied to the CP injury (Jaspers et al., 2009) and another one related with the upper extremity assessment by the use of robotic devices (Balasubramanian et al., 2012a), but to our knowledge, studies have not been found in relation to kinematic metrics for the upper extremity assessment regardless of the technology or device used for registering kinematic data and the neurological injury studied. Several technologies have been used for registering human movement. All these technologies are applied in clinical settings but the photogrammetry (opto-electronic systems based on active or passive markers) is considered the gold standard technology in human movement analysis.

In the study performed by Zollo, metrics were classified into two groups according to metrics related to biomechanical characteristics and metrics related to movement aspects (Zollo et al., 2011). Within the review that we present, metrics found in literature analyze different movement characteristics or quality measures, which have been shown in Figs. $2 \mathrm{a}$ and $2 \mathrm{~b}$. Usually, the same metrics have been found in literature under different labels, so in this review metrics have been called in a different way in relation to the original articles.

The articles reviewed have treated the neurological injuries of stroke, $\mathrm{CP}$ and SCI. The injury most frequently analyzed has been stroke with more than $50 \%$ of the studies reviewed. Only three studies have been found in SCI. It's worth taking into account that this injury is less common and its prevalence is minor in the population.

In relation to the movement analyzed in the reviewed articles, in healthy people the most common movement analyzed was ADL, in 7 $(77.8 \%)$ of the studies in this population. Normally, these studies analyzed several ADLs with the aim of computing normative data in relation to kinematic variables (Murgia et al., 2010; Murphy et al., 2006; Namdari et al., 2012). However, in populations with neurological injury analyzed in this review, the most frequently analyzed movements were functional movements such as reaching movements, reach and grasp movements or drawing trajectories as circles or squares. This highlights the need of developing kinematic metrics for expressing the outcomes with respect to the upper extremity function during basic ADL. One of the limitations found after the performed review is the lack of standardization between research groups in relation to the movements analyzed. This fact prevents the comparison between the outcomes of different studies.

The movement characteristic most frequently studied has been the smoothness in terms of the peak number in the hand velocity profile during the movement (Butler et al., 2010; Cacho et al., 2011; Chang et al., 2005; Fasoli et al., 2002; Kamper et al., 2002; Merlo et al., 2013; Murphy et al., 2011, 2013). However, one study has shown that this metric couldn't be robust against noise or other factors, proposing an analysis of the movement smoothness based on the Fourier Transform (Balasubramanian et al., 2012a). The accuracy during a movement has been analyzed along the trajectory of the hand (Colombo et al., 2008; Zollo et al., 2011) or around the target in reaching and grasping movements (Lang et al., 2005). The classification of the metrics into different groups related with quality or movement characteristics (Figs. 2a and 2b) must be analyzed from experimental data by means of a factor analysis. Recently, Merlo et al. have proposed a set of metrics through the use of a robotic device and have performed a factor analysis for classifying metrics into categories according to similar aspects of motor task. In this study, metrics were classified into three groups: accuracy, velocity and smoothness (Merlo et al., 2013). A similar study was previously performed and two factors were found between the kinematic variables analyzed (Murphy et al., 2011). Moreover, a correlation analysis was performed with the aim of analyzing the relation between different metrics. This analysis is interesting because metrics proposed could measure the same motor aspect and could offer redundant information in relation to the motor performance. 
In a recent study, Balasubramanian exposed several properties that a smoothness measure must accomplish with the aim of this measure may be useful (Balasubramanian et al., 2012b), but these properties could be applied to all kinematic metrics found in literature: (i) monotonic response: an increase in the metrics must always have associated a change in the movement characteristic that represent in the same direction, positive or negative. This author exposed that a decrease in smoothness is accompanied by an increase in submovement number and the time interval between them (Balasubramanian et al., 2012b); (ii) robustness: metrics must have consistency between repeated measures, because this metrics must be robust against noise and other environmental factors; (iii) sensitive to change: metrics must detect changes in the upper extremity motor performance and (iv) dimensionless: the authors exposed that, concretely, a smoothness measure must be dimensionless because the smoothness depends on the wave form of the velocity or acceleration profile and not on the amplitude and duration of the curve (Balasubramanian et al., 2012b).

Several studies have tried to analyze the validity of the kinematic metrics proposed, analyzing the correlation between metrics and clinical scales (Celik et al., 2010; Colombo et al., 2008; Osu et al., 2011; Van der Heide et al., 2005), but for the use of metrics as evaluative measures more effort must be performed in the analysis of aspects such as reproducibility and sensitivity to the change. Recently, a study has analyzed the sensitivity to the change and has demonstrated that metrics such as the movement time, smoothness and trunk range of motion have the ability of detecting improvements in the upper extremity function after the first three months after stroke (Murphy et al., 2013). To our knowledge similar studies have not been found in $\mathrm{SCl}$ or $\mathrm{CP}$ populations.

\section{Conclusions}

The translation of the information provided by means of kinematic studies into clinical interpretations must be further studied. The first findings have been found in stroke people mostly during reaching movements. After this review study, our challenge is the development of objective metrics related to the upper extremity dexterities and abilities, which in conjunction with clinical scales, could be applied to the functional evaluation of the upper extremity during the performance of activities of daily living. Our research center is specialized in SCI treatment, so the first experience will be applied to a population with cervical SCI. However, people with the upper extremity movement disorders after any neurological injury is the target population. On the other hand further research must be performed with respect to the analysis of the relation between all kinematic metrics and the validation for their possible use in a clinical setting.

\section{Conflict of interests}

The authors declare that they do not have significant financial, professional or personal interests that might have influenced the performance or presentation of the work describe in this manuscript.

\section{Acknowledgments}

This work has been supported by grant CSD2009-00067 CONSOUDER INGENIO 2010.

Authors' contributions have been classified into the following tasks:

- Article concept and design: Ana de los Reyes-Guzmán, Angel GilAgudo, Félix Monasterio-Huelin, Diego Torricelli.

- Literature search: Ana de los Reyes-Guzmán, Félix MonasterioHuelin, Iris Dimbwadyo-Terrer.

- Analysis and interpretation of published work: Ana de los ReyesGuzmán, Iris Dimbwadyo-Terrer, Fernando Trincado-Alonso.

- Drafting of manuscript: Ana de los Reyes-Guzmán, Iris DimbwadyoTerrer.
- Critical revision of manuscript for important intellectual content: Angel Gil-Agudo, Félix Monasterio-Huelin, Diego Torricelli.

- Article coordination: Angel Gil-Agudo.

\section{References}

Aizawa, J., Masuda, T., Koyama, T., Kojilsozaki, K.N., Okawa, A., Morita, S., 2010. Three dimensional motion of the upper extremity joints during various activities of daily living. J. Biomech. 43, 2915-2922.

Balasubramanian, S., Melendez-Calderon, A, Burdet, E, 2012a. A robust and sensitive metric for quantifying movement smoothness. IEEE Trans. Biomed. Eng. 59 (8), 2012

Balasubramanian, S., Colombo, R., Sterpi, I., Sanguineti, V., Burdet, E., 2012b. Robotic assessment of upper limb motor function after stroke. Robotics.

Bosecker, C., Dipietro, L., Volpe, B., Krebs, H.I., 2010. Kinematic robot-based evaluation scales and clinical counterparts to measure upper limb motor performance in patients with chronic stroke. Neurorehabil. Neural Repair 24 (1), 62-69.

Boyce, W., Gowland, C., Rosenbaum, P., Lane, M., Plews, N., Goldsmith, C., Russell, D., 1991. Measuring quality of movement in cerebral palsy: a review of instruments. Phys. Ther. 71, 813-819

Burdet, E., Milner, T., 1998. Quantization of human motions and learning of accurate movements. Biol. Cybern. 78, 307-318.

Butler, E.E., Ladd, A.L., Louie, S.A., LaMont, L.E., Wong, W., Rose, J., 2010. Temporal-spatial parameters of the upper limb during a reach \& grasp cycle for children. Gait Posture 32, 301-306.

Cacho, E.W.A., de Oliveira, R., Ortolan, R.L., Varoto Jr., R., A.C., 2011. Upper Limb Assessment in Tetraplegia: Clinical, Functional and Kinematic Correlations. Wolters Kluwer Health. Lippincott Williams \& Wilkins.

Catz, A., Itzkovich, M., Agranov, E., Ring, H., Tamir, A., 1997. SCIM-spinal cord independence measure: a new disability scale for patients with spinal cord lesions. Spinal Cord 35 (12), 1997.

Celik, O., O'Malley, M.K., Boake, C., Levin, H.S., Yozbatiran, N., Reistetter, T.A., 2010. IEEE Trans. Neural Syst. Rehabil. 18 (4).

Chang, J.J., Wu, T.I., Wu, W.L., Su, F.C., 2005. Kinematical measure for spastic reaching in children with cerebral palsy. Clin. Biomech. 20, 381-388.

Colombo, R., Pisano, F., Micera, S., Mazzone, A., Delconte, C., Carrozza, M.C., Dario, P., Minuco, G., 2005. Robotic techniques for upper limb evaluation and rehabilitation of stroke patients. IEEE Trans. Neural Syst. Rehabil. 13 (3).

Colombo, R., Pisano, F., Mazzone, A., Delconte, C., Micera, S., Carrozza, M.C., Dario, P. Minuco, G., 2007. Design strategies to improve patient motivation during robotaided rehabilitation. J. Neuroeng. Rehabil. 4, 3.

Colombo, R., Pisano, F., Micera, S., Mazzone, A., Delconte, C., Carrozza, M., Dario, P., Minuco, G., 2008. Assessing mechanisms of recovery during robot-aided neurorehabilitation of the upper limb. Neurorehabil. Neural Repair 22, 50.

Colombo, R., Sterpi, I., Mazzone, A., Delconte, C., Pisano, F., 2012. Taking a lesson from patients' recovery strategies to optimize training during robot-aided rehabilitation. IEEE Trans. Neural Syst. Rehabil. 20 (3).

Culmer, P.R., Levesley, M.C., Mon-Williams, M., Williams, J.H.G., 2009. A new tool for assessing human movement: the kinematic assessment tool. J. Neurosci. Methods $184,184-192$.

de los Reyes-Guzmán, A., Gil-Agudo, A., Peñasco-Martín, B., Solís-Mozos, M., del AmaEspinosa, A., Pérez-Rizo, E., 2010. Kinematic analysis of the daily activity of drinking from a glass in a population with cervical spinal cord injury. J. Neuroeng. Rehabil. 7 , 41.

Dipietro, L., Krebs, H.I., Fasoli, S.E., Volpe, B.T., Stein, J., Bever, C., Hogan, N., 2007. Changing motor synergies in chronic stroke. J. Neurophysiol. 98, 757-768.

Ellis, D., Sukal, T., DeMott, T., Dewald, J.P.A., 2008. Augmenting clinical evaluation of hemiparetic arm movement with a laboratory-based quantitative measurement of kinematics as a function of limb loading. Neurorehabil. Neural Repair 22 (4), 321-329.

Fasoli, S.E., Trombly, C.A., Tickle-Degnen, L., Verfaellie, M.H., 2002. Effect of instructions on functional reach in persons with and without cerebrovascular accident. Am. J. Occup. Ther. 56, 380-390.

Fitoussi, F., Diop, A., Maurel, N., Laassel, E.M., Pennecot, G.F., 2006. Kinematic analysis of the upper limb: a useful tool in children with cerebral palsy. J. Pediatr. Orthop. B $15,247-256$.

Fitts, P.M., 1954. The information capacity of the human motor system in controlling the amplitude of movement. J. Exp. Psychol. 47, 381-391.

Fugl-Meyer, A.R., Jaasko, L., Leyman, I., Olsson, S., Steglind, S., 1974. The post-stroke hemiplegic patient. 1. A method for evaluation of physical performance. Scand. J. Rehabil. Med. 7 (1), 13-31

Hogan, N., Sternad, D., 2009. Sensitivity of smoothness measures to movement duration, amplitude, and arrests. J. Motor Behav. 41 (6), 529-534.

Jaspers, E., Desloovere, K., Bruyninckx, H., Katrijn Klingels, G.M., Feys, H., 2009. Review of quantitative measurements of upper limb movements in hemiplegic cerebral palsy. Gait Posture 30, 395-404.

Jaspers, E., Desloovere, K., Bruyninckx, H., Klingels, K., Molenaers, G., Aertbeliën, E., Van Gestel, L., Feys, H., 2011. Three-dimensional upper limb movement characteristics in children with hemiplegic cerebral palsy and typically developing children. Res. Dev. Disabil. 32, 2283-2294.

Jebsen, R.H., Taylor, N., Trieschmann, R.B., Trotter, M.J., Howard, L.A., 1969. An objective and standardized test of hand function. Arch. Phys. Med. Rehabil. 311-319.

Kamper, D.G., McKenna-Cole, A.N., Kahn, L.E., Reinkensmeyer, D.J., 2002. Alterations in reaching after stroke and their relation to movement direction and impairment severity. Arch. Phys. Med. Rehabil. 83, 702-707. 
Keith, R.A., 1987. The functional independence measure: a new tool for rehabilitation. Adv. Clin. Rehabil. 2, 6-18.

Kim, K., Song, W.K., Lee, J., Lee, H.Y., Park, D.S., Ko, B.W., Kim, J., 2014. Kinematic analysis of upper extremity movement during drinking in hemiplegic subjects. Clin. Biomech. 29 (3), 248-256

Klotz, M.C.M., Kost, L., Braatz, F., Ewerbeck, V., Heitzmann, D., Gantz, S., Dreher, T., Wolf, S. I., 2013. Motion capture of the upper extremity during activities of daily living in patients with spastic hemiplegic cerebral palsy. Gait Posture 38, 148-152.

Lang, C.E., Wagner, J.M., Bastian, A.J., Hu, Q., Edwards, D.F., Sahrmann, S.A., Dromerick, A. W., 2005. Deficits in grasp versus reach during acute hemiparesis. Exp. Brain Res. 166, 126-136.

Lang, C.E., Wagner, J.M., Edwards, D.F., Sahrmann, S.A., Dromerick, A.W., 2006. Recovery of grasp versus reach in people with hemiparesis poststroke. Neurorehabil. Neural Repair 20, 444-454.

Lum, P.S., Mulroy, S., Amdur, R.I.., Requejo, P., Prilutsky, B.I., Dromerick, A.W., 2009. Gains in upper extremity function after stroke via recovery or compensation: potential differential effects on amount of real-world limb use. Top. Stroke Rehabil. 16 (4), 237-253.

Magermans, D., Chadwick, E., Veeger, H., van der Helm, F., 2005. Requirements for upper extremity motions during activities of daily living. Clin. Biomech. 20, 591-599.

Mahoney, F.I., 1965. Functional evaluation: the Barthel index. Md. State Med. J. 14, 61-65

Merlo, A., Longhi, M., Giannotti, E., Prati, P., Giacobbi, M., Ruscelli, E., Mancini, A., Ottaviani, M., Montanari, L., Mazzoli, D., 2013. Upper limb evaluation with robotic exoskeleton. Normative values for indices of accuracy, speed and smoothness. NeuroRehabilitation $33,523-530$.

Murgia, A., Kyberd, P., Barnhill, T., 2010. The use of kinematic and parametric information to highlight lack of movement and compensation in the upper extremities during activities of daily living. Gait Posture 31, 300-306.

Murphy, M.A., Sunnerhagen, K.S., Johnels, B., Will_en, C., 2006. Three-dimensional kinematic motion analysis of a daily activity drinking from a glass: a pilot study. J. Neuroeng. Rehabil. 3, 18

Murphy, M.A., Will_en, K.S., C., Sunnerhagen, K.S., 2011. Kinematic variables quantifying upper extremity performance after stroke during reaching and drinking from a glass. Neurorehabil. Neural Repair 25 (1), 71-80.

Murphy, M.A., Will en, C., Sunnerhagen, K.S., 2013. Responsiveness of upper extremity kinematic measures and clinical improvement during the first three months after stroke. Neurorehabil. Neural Repair 27,844.

Nakayama, H., Jorgensen, H., Raaschou, H., Olsen, T., 1994. Compensation in recovery of upper extremity function after stroke: the Copenhagen stroke study. Arch. Phys. Med. Rehabil. 75, 852-857.

Namdari, S., Yagnik, G., Ebaugh, D.D., Nagda, S., Ramsey, M.L., Williams Jr., G.R., Mehta, S., 2012. Defining functional shoulder range of motion for activities of daily living. J. Shoulder Elb. Surg. 21 (9), 1177-1183.

Osu, R., Ota, K., Fujiwara, T., Otaka, Y., Kawato, M., Liu, M., 2011. Quantifying the quality of hand movement in stroke patients through three-dimensional curvature. J. Neuroeng. Rehabil. 8 (26), 62.

Parker, V., Wade, D., Hewer, R.L., 1986. Loss of arm function after stroke: measurement frequency, and recovery. Int. Rehabil. Med. 8, 69-73.
Petuskey, K., Bagley, A., Abdala, E.. James, M., Rab, G., 2007. Upper extremity kinematics during functional activities: three-dimensional studies in a normal pediatric population. Gait Posture 25, 573-579.

Rand, M., Shimansky, Y., Stelmach, G., Bracha, V., Bloedel, J., 2000. Effects of accuracy constraints on reach-to-grasp movements in cerebellar patients. Exp. Brain Res. 135, $179-188$.

Rohrer, B., Fasoli, S., Krebs, H.I., Hughes, R., Volpe, B., Frontera, W.R., Stein, J., Hogan, N., 2002. Movement smoothness changes during stroke recovery. J. Neurosci. 22 (18), 8297-8304.

Rönnquist, L., Rösblad, B., 2007. Kinematic analysis of unimanual reaching and grasping movements in children with hemiplegic cerebral palsy. Clin. Biomech. 22, $165-175$.

Thies, S.B., Tresadern, P.A., Kenney, L.P., Smith, J., Howard, D., Goulermas, J.Y., Smith, C., Rigby, J., 2009. Movement variability in stroke patients and controls performing two upper limb functional tasks: a new assessment methodology. J. Neuroeng. Rehabil. 6, 2 .

Trombly, C.A., Wu, C.Y., 1999. Effect of rehabilitation tasks on organization of movement after stroke. Am. J. Occup. Ther. 53, 333-344.

van Andel, C.J., Wolterbeek, N., Doorenbosch, C.A., Veeger, D.H., Harlaar, J., 2008. Complete 3D kinematics of upper extremity functional tasks. Gait Posture 27, 120-127.

Van der Heide, J.C., Fock, J.M., Otten, B., Stremmelaar, E., Hadders-Algra, M., 2005. Kinematic characteristics of reaching movements in preterm children with cerebral palsy. Pediatr. Res. 57, 883-889.

van Tuijl, J., Janssen-Potten, Y., Seelen, H., 2002. Evaluation of upper extremity motor function tests in tetraplegics. Spinal Cord 40, 51-64.

Vergaro, E., Casadio, M., Squeri, V., Giannoni, P., Morasso, P., Sanguineti, V., 2010. Self adaptive robot training of stroke survivors for continuous tracking movements. J. Neuroeng. Rehabil. 7, 13

Volman, M.C.J., Wijnroks, A.I., Vermeer, A., 2002. Effect of task context on reaching performance in children with spastics hemiparesis. Clin. Rehabil. 16, 684 .

Wade, D.T., 1992. Measurement in neurological rehabilitation. Curr. Opin. Neurol. 5 (5), 682-686.

Wagner, J.M., Lang, C.E., Sahrmann, S.A., Edwards, D.F., Dromerick, A.W., 2007. Sensorimotor impairments and reaching performance in subjects with poststroke hemiparesis during the first few months of recovery. Phys. Ther. 87, 751-765.

Wagner, J.M., Rhodes, J.A., Patten, C., 2008. Reproducibility and minimal detectable change of three-dimensional kinematic analysis of reaching tasks in people with hemiparesis after stroke. Phys. Ther. 88, 652-663.

Yang, N., Zhang, M., Huang, C., Jin, D., 2002. Motion quality evaluation of upper limb target reaching movements. Med. Eng. Phys. 24 (2), 115-120.

Zariffa, J., Kapadia, N., Kramer, J.L.K., Taylor, P., Alizadeh-Meghrazi, M., Zivanovic, V., Albisser, U., et al., 2012. Relationship between clinical assessments of function and measurements from an upper-limb robotic rehabilitation device in cervical spinal cord injury. IEEE Trans. Neural Syst. Rehabil. 20 (3).

Zollo, L., Rossini, L., Bravi, M., Magrone, G., Sterzi, S., Guglielmelli, E., 2011. Quantitative evaluation of upper-limb motor control in robot-aided rehabilitation. Med. Biol. Eng. Comput. 49, 1131-1144. 